\title{
Kapazitive Elastomersensoren zur Detektion von Fingerdrücken
}

\author{
Holger Böse, Deniz Ocak, Stephan Wirthmann, Johannes Ehrlich \\ Fraunhofer-Institut für Silicatforschung, Neunerplatz 2, 97082 Würzburg, Germany, \\ E-mail: holger.boese@isc.fraunhofer.de
}

\begin{abstract}
Zusammenfassung
In dem Beitrag werden flexible, weiche und hoch empfindliche kapazitive Drucksensoren beschrieben, die zur Detektion von Fingerdrücken eingesetzt werden können. Die Sensoren bestehen aus parallel angeordneten und miteinander verbundenen elektrisch isolierenden und leitfähigen Schichten aus Siliconelastomeren. Die äußeren Schichten weisen auf der Innenseite profilierte Oberflächen auf, zwischen denen eine innere planare Elastomerfolie beim Zusammendrücken des Sensors gedehnt wird. Mit steigendem Druck ändert sich die elektrische Kapazität zwischen den leitfähigen Schichten kontinuierlich, woraus die hohe Sensorempfindlichkeit resultiert. Zwei beispielhafte Anwendungen werden gezeigt, in denen die neuen Drucksensoren zur Messung von Fingerdrücken genutzt werden können. In der einen Anwendung werden derartige Drucksensoren in einen Handschuh integriert. Beim Greifen eines Gegenstandes lassen sich damit die mit den Fingern ausgeübten Greifkräfte erfassen. In der zweiten Anwendung werden die Drucksensoren auf einer Bedienoberfläche genutzt, um die mit einem Finger ausgeübte Druckkraft zu messen. Über die Stärke der Druckkraft kann damit eine technische Funktion wie beispielsweise die Helligkeit einer Lampe gesteuert werden.
\end{abstract}

Keywords: Drucksensoren, flexible Elastomersensoren, Fingerdruck, Greifkraft, Bedienelement

\section{Einleitung}

Wenn Menschen mechanische Tätigkeiten verrichten, tun sie dies in der Regel mit ihren Händen. Die menschliche Hand kann mit den einzelnen Fingern wohl dosiert Druck ausüben, damit Gegenstände greifen und sie festhalten. Ebenso kann mit einem Finger ein definierter Druck auf eine Oberfläche erzeugt werden, um beispielsweise eine technische Funktion wie die Betätigung eines Motors zu steuern.

Um den mit den Fingern ausgeübten Druck quantitativ zu detektieren, sind geeignete Sensoren notwendig. Da die Finger wie auch die ganze Hand weich und nachgiebig sind, sollten auch die Sensoren, die den Druck erfassen, entsprechende mechanische Eigenschaften aufweisen. Im Idealfall sind sie weich, flexibel und verformbar und passen sich der Kontur der Haut an.

Bereits heute gibt es eine Vielzahl von Drucksensoren mit unterschiedlichen Messprinzipen, die kommerziell erhältlich sind. Dabei handelt es sich jedoch meist um harte und mechanisch starre Sensoren, die nicht die gewünschte Flexibilität und Verformbarkeit aufweisen.
Am weitesten verbreitet sind Dehnmessstreifen, die bereits bei geringen Verformungen ihren elektrischen Widerstand merklich verändern. Auch piezoelektrische Sensoren können schon bei kleinen Verformungen deutliche Signale liefern, doch reagieren sie nur dynamisch, während das Signal bei statischer Belastung mit der Zeit abfällt. Diese Sensoren sind jedoch nicht weich und flexibel genug, um sich der Haut anzupassen und damit für Messungen von Fingerdruckkräften weniger geeignet.

Dielektrische Elastomersensoren (DES) sind dagegen vergleichsweise sehr weich, hoch flexibel und darüber hinaus dehnbar. Sie eignen sich damit besonders zur Messung von Dehnungen in ein oder zwei Dimensionen.

Abb. 1 zeigt das zugrundeliegende Messprinzip solcher Dehnungssensoren. Eine stark dehnbare Elastomerfolie ist beidseitig mit ebenfalls dehnbaren Elektroden beschichtet. Bei dieser Konfiguration handelt es sich um einen verformbaren elektrischen Plattenkondensator. Bei einer uni- oder auch bidirektionalen Dehnung erhöht sich die Fläche der Elastomerfolie, während die Dicke abnimmt. Beide Effekte tragen zur Erhöhung 
der Kapazität bei, sodass durch einfache Kapazitätsmessungen der Dehnungszustand der Elastomerfolie erfasst wird [1].

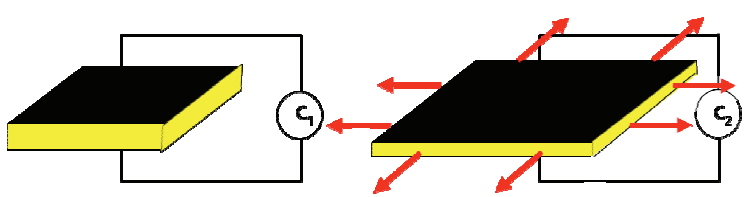

Abb. 1. Erhöhung der elektrischen Kapazität bei Dehnung der dielektrischen Elastomerfolie

Solche dielektrischen Elastomersensoren sind besonders zur Messung von hohen Dehnungen sehr gut geeignet und bereits kommerziell verfügbar [2]. Für die Messung von Druckbelastungen weisen sie dagegen nur eine geringe Empfindlichkeit auf, da sich insbesondere auf wenig nachgiebigen Untergründen die Kapazität infolge der geringen Verformbarkeit nur wenig verändert. In den letzten Jahren wurden verschiedene Ansätze auch für flexible kapazitive Drucksensoren aufgezeigt [3-7]. In den Sensoren werden teilweise geschäumte Folien als Dielektrikum verwendet, die sich bei Druckausübung komprimieren lassen $[4,6,7]$. Auch bei diesen Sensoren kommt ein kapazitives Messprinzip zum Einsatz.

Auf der Basis von dielektrischen Elastomeren wurden kürzlich neuartige Drucksensormatten entwickelt, die eine sehr hohe Empfindlichkeit aufweisen [8-11]. Die Sensormatten bestehen aus einem Schichtaufbau aus zwei profilierten Außenfolien aus Elstomer, zwischen denen sich eine ebene Elastomerfolie befindet. Durch vielfältige Variationsmöglichkeiten bei den verwendeten Materialien und am Sensordesign lässt sich die Kennlinie des Sensors in einem weiten Bereich einstellen.

Im Folgenden sollen zunächst der Sensoraufbau in verschiedenen Versionen dargestellt und das zugrundeliegende Messprinzip dieser flexiblen Drucksensoren erläutert werden. Im zweiten Teil werden mögliche Anwendungen beschrieben, bei denen die neuen Drucksensoren besonders vorteilhaft zur Messung von Fingerdrücken eingesetzt werden.

\section{Sensoraufbau und Messprinzip}

Der Aufbau von flexiblen Drucksensoren auf Basis dielektrischer Elastomere zeichnet sich durch eine hohe Variabilität aus. Eine Version, die das zugrundeliegende Messprinzip schematisch verdeutlicht, ist in Abb. 2 dargestellt [9]. Dabei befindet sich eine dielektrische Elastomerfolie mit drei
Elektrodenschichten zwischen zwei profilierten Elastomerfolien mit Wellenform.
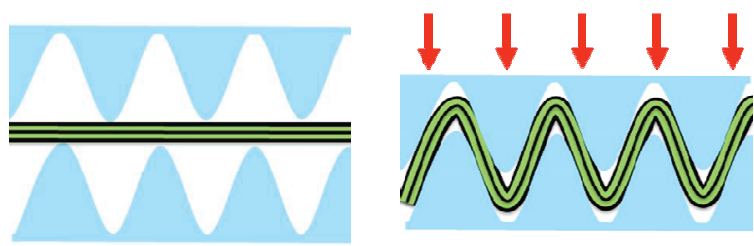

\section{Profil Dielektrikum -Elektrode \\ Abb. 2. Schematischer Aufbau von Sensormatte mit Wellenprofilen und drei Elektrodenschichten auf der Zwischenfolie}

Beim Zusammendrücken dieser Sensormatte dringen die Wellenprofile ineinander ein und verformen die dazwischen liegende planare Elastomerfolie, die in der Länge gedehnt wird, wodurch sich ihre Kapazität erhöht. Die Druckbelastung auf den Sensor wird somit in eine Zugbelastung auf die Zwischenfolie konvertiert. Durch die zwei Dielektrikumsschichten zwischen den drei Elektrodenschichten in der Zwischenfolie wird die Kapazität bei gleicher Folienfläche verdoppelt. Jedes Wellenprofil hat eine Dicke von etwa 4 $\mathrm{mm}$. Damit weist die gesamte Sensormatte eine Dicke von etwa $8 \mathrm{~mm}$ auf.

In einem zweiten Ansatz für eine Sensormatte wurde ein verändertes Design genutzt, bei dem die zwei äußeren Elektrodenschichten von der Zwischenfolie auf die Wellenprofile verlagert wurden (Abb. 3). Nur die dritte Elektrodenschicht verbleibt im Innern der Elastomerzwischenfolie. Diese scheinbar kleine Veränderung der Elektrodenposition hat große Auswirkungen auf die Kennlinie der Sensormatte, wie in Abb. 4 zu erkennen ist.
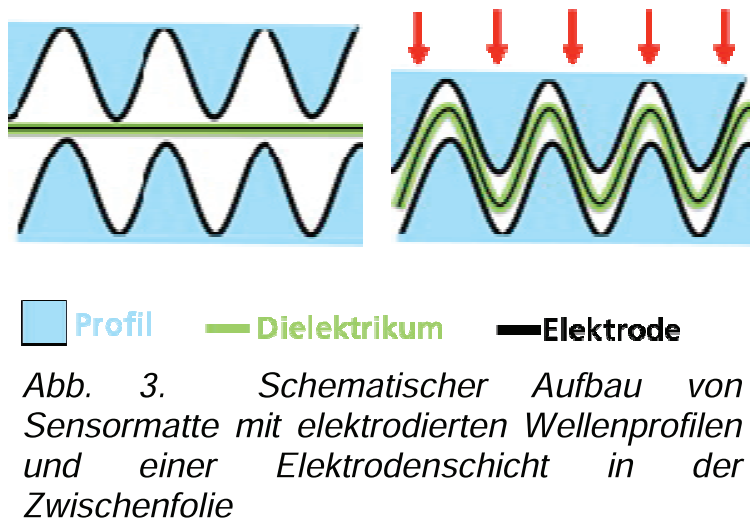

Während die erste Version der Sensormatte ohne Elektroden auf den Profilen ohne Belastung bereits eine relativ hohe Kapazität von etwa 6,5 nF aufweist, startet die Kapazität der zweiten Version mit Profilelektroden bei einem sehr geringen Wert von ca. 0,5 nF. Die 
Kapazität beider Sensormatten wächst mit steigender Belastung kontinuierlich an und erreicht bei $1000 \mathrm{~N}$ in beiden Fällen einen Wert von etwa $13 \mathrm{nF}$. Die maximale Belastung von $1000 \mathrm{~N}$ entspricht bei den beiden Sensormatten mit einer Fläche von ca. $10 \mathrm{~cm}$ x $10 \mathrm{~cm}$ einem Druck von $10 \mathrm{~N} / \mathrm{cm}^{2}$ bzw. 1 bar bzw. $100 \mathrm{kPa}$.

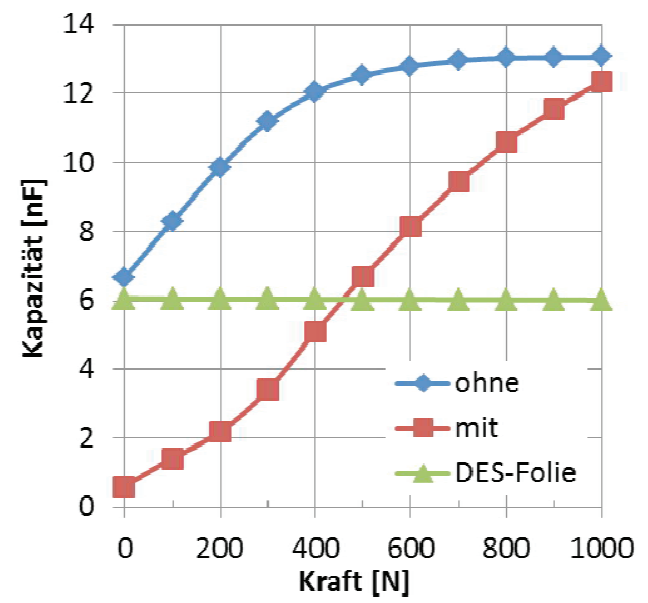

Abb. 4. Kapazitätsänderung von Sensormatten mit Wellenprofilen ohne bzw. mit Profilelektroden sowie von DES-Folie bei steigender Druckbelastung

Die erste Version der Sensormatte ohne Profilelektroden erreicht damit eine Steigerung der Kapazität von etwa $100 \%$, wobei die wesentliche Kapazitätssteigerung im ersten Teil der Belastungserhöhung erfolgt. Bei der zweiten Version der Sensormatte mit Profilelektroden steigt die Kapazität dagegen um mehr als einen Faktor 10. Dabei verläuft der Anstieg in weiten Bereichen nahezu linear. Diese Sensormatte erreicht damit eine extrem hohe Empfindlichkeit im hier untersuchten Druckbereich.

In Abb. 4 ist zum Vergleich auch der Kapazitätsverlauf einer reinen DES-Folie ohne Profile (Zwischenfolie der ersten Version der Sensormatte) im selben Belastungsbereich dargestellt. Die grüne Kurve bestätigt sehr deutlich, dass die gegenüber Zugbelastungen empfindliche DES-Folie bei Druckbelastungen absolut unempfindlich reagiert und keine messbare Kapazitätssteigerung zeigt.

Das in Abb. 2 und 3 gezeigte Wellenprofil stellt nur eine mögliche Profilform dar. Die zueinander parallelen Wellen ziehen sich durch die gesamte Sensormatte und geben ihr eine Vorzugsrichtung, die zu einer anisotropen Biegesteifigkeit führt. Eine andere Profilform, die ebenfalls Gegenstand von Untersuchungen war, ist eine Stegform. Auch hier verlaufen die Stege parallel zueinander und erzeugen ebenfalls eine Anisotropie, doch sind die Stege wesentlich flacher, sodass die entsprechende Sensormatte eine Dicke von nur noch etwa 3 $\mathrm{mm}$ und damit eine deutlich geringere Biegesteifigkeit aufweist.

Eine weitere Profilform besteht in einem Noppenprofil, bei dem zylinderförmige Noppen in einer zweidimensionalen Struktur angeordnet sind. Abb. 5 zeigt den schematischen Aufbau einer solchen Sensormatte mit der Zwischenfolie zwischen den beiden mit Elektroden beschichteten Noppenprofilen.

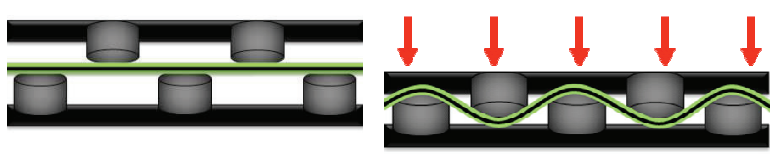

L Profil - Dielektrikum -Elektrode

Abb. 5. Schematischer Aufbau von Sensormatte mit elektrodierten Noppenprofilen und einer Elektrodenschicht in der Zwischenfolie

In Abb. 6 ist ein derartiges Noppenprofil mit einer darauf aufgebrachten Elektrodenschicht dargestellt. Man erkennt darauf das zweidimensionale Noppenmuster mit Zwischenräumen.

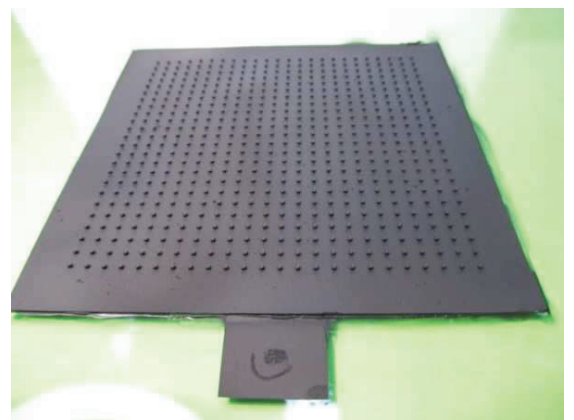

Abb. 6. Noppenprofil mit Elektrodenschicht
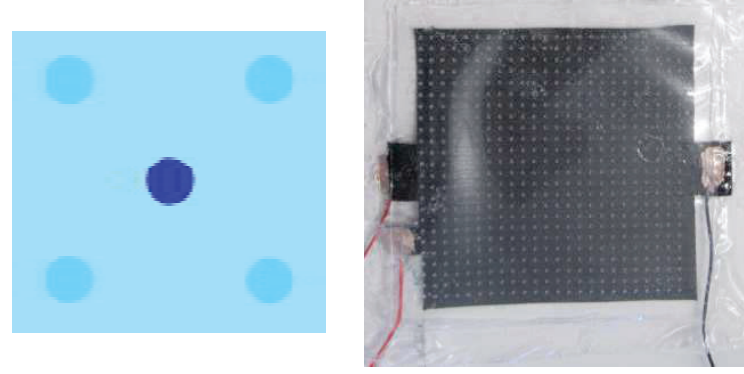

Abb. 7. Schema der Anordnung der Noppen auf den beiden Profilen zueinander (links) und komplette Sensormatte mit Noppenprofilen (rechts)

Abb. 7 links zeigt schematisch die Anordnung der beiden Noppenprofile zueinander. Die 
Noppen des einen Profils greifen mittig in die Zwischenräume des anderen Noppenprofils und verformen dabei die Zwischenfolie. In Abb. 7 rechts ist eine komplette Sensormatte mit Noppenprofilen dargestellt. Auch hier beträgt die Gesamtdicke der Sensormatte etwa $3 \mathrm{~mm}$. Die Flexibilität ist wegen des zweidimensionalen Musters des Profils am höchsten.

Auch die Profilform hat einen erheblichen Einfluss auf den Verlauf der Kennlinie der Sensormatte. Abb. 8 zeigt den Vergleich der drei Sensormatten mit Wellen-, Steg- und Noppenprofilen. Bei allen drei Typen von Sensormatten sind auf den Profiloberflächen Elektrodenschichten aufgebracht. Man erkennt, dass die Sensormatte mit den Wellenprofilen den größten absoluten Kapazitätsanstieg aufweist. Die Sensormatte mit den Noppenprofilen zeigt jedoch ebenfalls einen großen relativen Anstieg und ist zudem sehr flexibel.

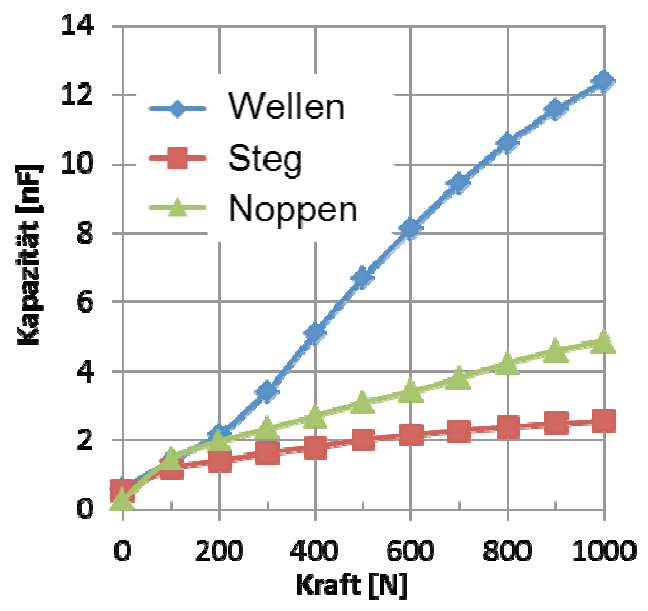

$A b b$. 8. Kapazitätsänderung von verschiedenen Sensormatten mit Wellenprofilen, mit Stegprofilen und mit Noppenprofilen, jeweils mit Profilelektroden, bei steigender Druckbelastung

Die dargestellten Sensormatten wurden vollständig aus Siliconelastomer hergestellt. Dabei kann die Härte der profilierten Folien als auch der ebenen Zwischenfolie durch die Art des verwendeten Silicons in einem weiten Bereich verändert werden. Insbesondere der Grad der chemischen Vernetzung des Silicons bestimmt die mechanische Härte des Elastomers. Auch dies hat einen erheblichen Einfluss auf die Kennlinie der Sensormatte.

Die Elektrodenschichten auf den Profilen sowie in den Zwischenfolien bestehen ebenfalls aus Siliconelastomer. Die Leitfähigkeit wird dabei durch die Einarbeitung von leitfähigen Partikeln wie Ruß erzeugt.
Zur Aufnahme der Kennlinien der Kapazität in Abhängigkeit von der Belastungskraft wurden die Sensormatten in einer mechanischen Prüfmaschine mit steigender Kraft zusammengedrückt. Bei jeder Kraftstufe wurde die Kapazität mit einem LCR-Meter gemessen.

\section{Detektion von Fingerdrücken}

Die Nutzung der flexiblen Drucksensoren auf Basis dielektrischer Elastomere für die Messung von Fingerdrücken wird im Folgenden anhand von zwei möglichen Anwendungen diskutiert. Bei der ersten Anwendung wird ein Handschuh vorgestellt, der mit Drucksensoren an den Fingern ausgerüstet ist, sodass damit Greifkräfte gemessen werden können. Im zweiten Fall wird eine Bedienoberfläche beschrieben, bei der eine technische Funktion über die Druckstärke des Fingers gesteuert werden kann.

Abb. 9 zeigt eine erste Version eines Handschuhs, bei dem ein Drucksensor mit der passenden Größe außen auf dem Teil des Handschuhs aufgebracht ist, der über den Zeigefinger gezogen wird.
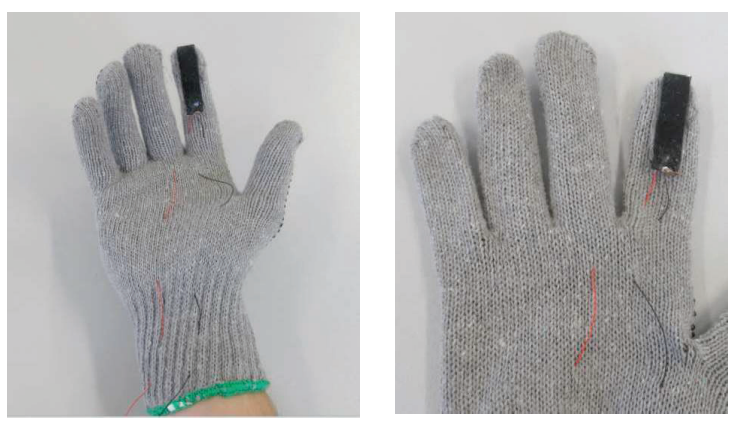

Abb. 9. Handschuh mit auf dem Zeigefinger aufgebrachtem Drucksensor

Um die Empfindlichkeit und Reproduzierbarkeit des Sensors auf dem Handschuh zu prüfen, wurden zunächst systematische Belastungsversuche durchgeführt. Dabei wurden verschiedene Gewichte (bis $500 \mathrm{~g}$ ) auf die Sensoroberfläche (ca. $5 \mathrm{~cm}^{2}$ ) auf dem Handschuh gelegt, worauf jeweils die Kapazität mit einem LCR-Meter gemessen wurde.

Abb. 10 zeigt die Ergebnisse dieser Untersuchungen. Neben der Kurve aus den Mittelwerten der Kapazitätsmessungen sind auch die Werte des um eine Standardabweichung reduzierten bzw. erhöhten Mittelwertes aufgetragen. Dabei wird die gute Reproduzierbarkeit in Form einer sehr geringen Streuung sichtbar. Außerdem weicht die Kurvenform in diesem Belastungsbereich bis $1 \mathrm{~N} / \mathrm{cm}^{2}$ bzw. $10 \mathrm{kPa}$ nicht wesentlich von einem linearen Verlauf $a b$, was bei Sensoren in der Regel erwünscht ist. 


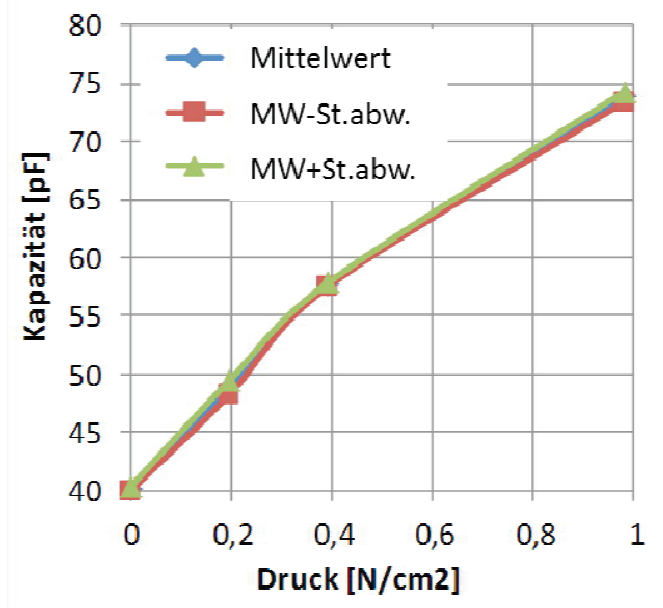

Abb. 10. Gemessene Abhängigkeit der Kapazität des Handschuhsensors vom Belastungsdruck: Mittelwert der Kapazität sowie um Standardabweichung reduzierter bzw. erhöhter Mittelwert

In Abb. 11 sind die Ergebnisse von zyklischen Belastungsversuchen dargestellt. Dabei wurde die Gewichtsbelastung dreimal bis $1,5 \mathrm{~kg}$ stufenweise gesteigert und wieder reduziert. Die maximale Belastung entspricht damit 3 $\mathrm{N} / \mathrm{cm}^{2}$ bzw. $30 \mathrm{kPa}$. Man erkennt hier ebenfalls eine sehr gute Reproduzierbarkeit und eine vernachlässigbare Hysterese.

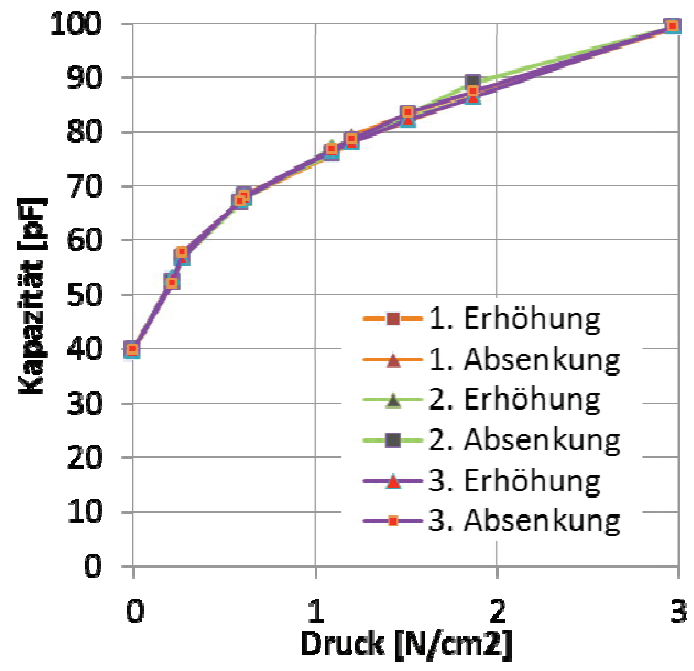

Abb. 11. Abhängigkeit der Kapazität des Handschuhsensors vom Belastungsdruck in drei Zyklen der Erhöhung und Absenkung der Belastung

Zur Prüfung des Kriechverhaltens der Sensoren wurden weitere Untersuchungen durchgeführt, bei denen eine konstante Belastung über eine längere Zeit aufrechterhalten wurde. Hierzu wurde ein Gewicht von
$500 \mathrm{~g}$ über 3 Stunden auf dem Sensor platziert. Abb. 12 zeigt den gemessenen Verlauf der Kapazität über der Zeit. Dabei wurde nach den 3 Stunden eine Kapazitätserhöhung von etwa $1 \%$ festgestellt. Damit wird deutlich, dass Kriechvorgänge unter diesen Bedingungen zu keiner merklichen Beeinträchtigung des Sensors führen.

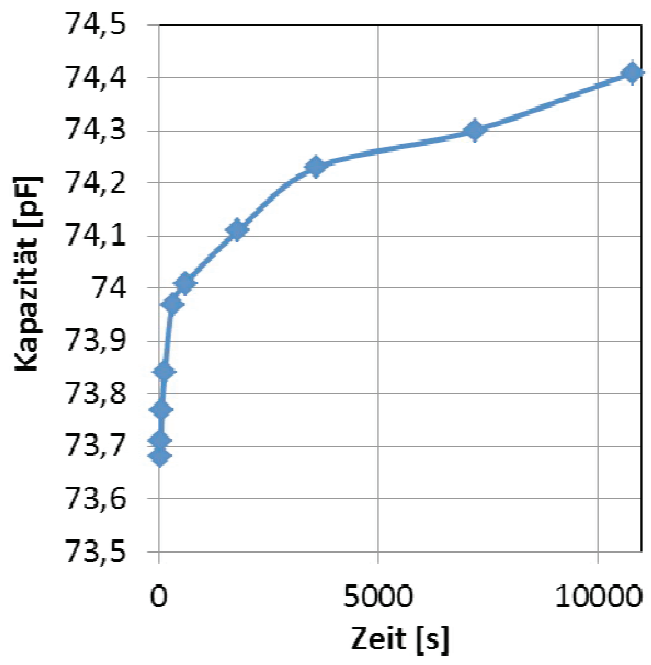

$A b b$. 12. Veränderung der Kapazität des Handschuhsensors bei konstanter Belastung mit $500 \mathrm{~g}$ über 3 Stunden

In Abb. 13 wird der praktische Einsatz des Handschuhsensors demonstriert. Der Einfachheit halber wurde hier ein Multimeter zur Kapazitätsanzeige verwendet, sodass die Messwerte nicht mit den zuvor mit dem LCRMeter gemessenen Daten vergleichbar sind. Im oberen Bild wird für den unbelasteten Sensor eine Kapazität von 15 pF angezeigt. Beim Greifen eines Schraubenschlüssels im mittleren Bild steigt die gemessene Kapazität auf 96 pF an. Wird schließlich ein Gewicht von $2 \mathrm{~kg}$ gegriffen, so steigt die Kapazität sogar bis auf $162 \mathrm{pF}$ an. Dies erlaubt eine deutliche Differenzierung der Greifkräfte beim Halten verschiedener Gegenstände.

Nach den Versuchen mit einem Handschuh mit nur einem Drucksensor wurde ein zweiter Handschuh mit insgesamt sieben Sensoren ausgerüstet. Davon befinden sich fünf Sensoren auf den verschiedenen Fingern und zwei Sensoren auf der inneren Handfläche. Abb. 14 zeigt den Handschuh mit den sieben Drucksensoren.

Da hier insgesamt sieben Kapazitätswerte gleichzeitig gemessen werden müssen, wurde eine dafür geeignete Elektronik in einer Box aufgebaut. Zur simultanen optischen Anzeige der Kapazitäten wurde die Box mit einem Display ausgestattet, das die Kapazitätswerte als Balken darstellt. Abb. 14 zeigt neben dem Handschuh auch die Box mit dem Display. 


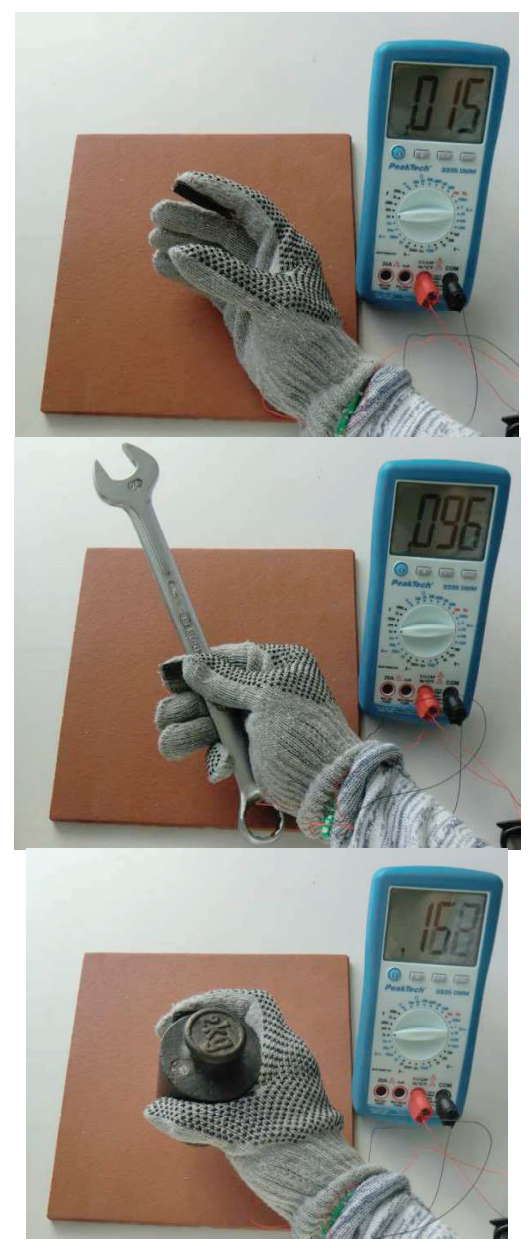

$A b b$. 13. Veränderung der Kapazität des Handschuhsensors bei verschiedenen Greifvorgängen: $15 \mathrm{pF}$ im unbelasteten Zustand (oben), 96 pF beim Greifen eines Schraubenschlüssels (Mitte) und 162 pF beim Greifen eines Gewichtes von 2 kg (unten)

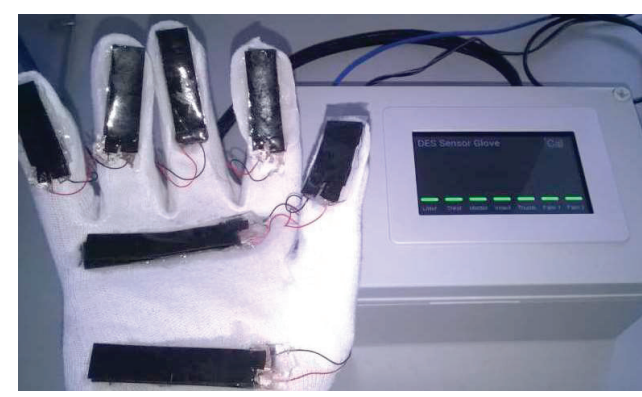

Abb. 14. Handschuh mit insgesamt sieben Drucksensoren und Elektronik-Box mit Display

Auch mit diesem Handschuh wurden praktische Versuche durchgeführt, bei denen verschiedene Tätigkeiten ausgeführt wurden. Um die Sensoren vor möglichen Beschädigungen zu schützen, wurde der
Handschuh mit den Sensoren mit einem weiteren Handschuh überzogen.

In Abb. 15 sind verschiedene Szenarien mit dem Handschuh mit den sieben Sensoren dargestellt. Im oberen Bild wirkt keine Belastung auf den Handschuh und die Sensoren. Werden Daumen und Zeigefinger zusammengedrückt, so wird dies anhand der zugehörigen Balken auf dem Display sichtbar. (mittleres Bild). Beim Halten eines Gegenstandes mit $2 \mathrm{~kg}$ Gewicht sind alle Finger und auch die Handfläche mehr oder weniger beteiligt (Bild unten). Eine solche HandschuhSensorik kann damit die Druckverteilung der Hand detailliert erfassen und so helfen, den Greifvorgang zu analysieren.

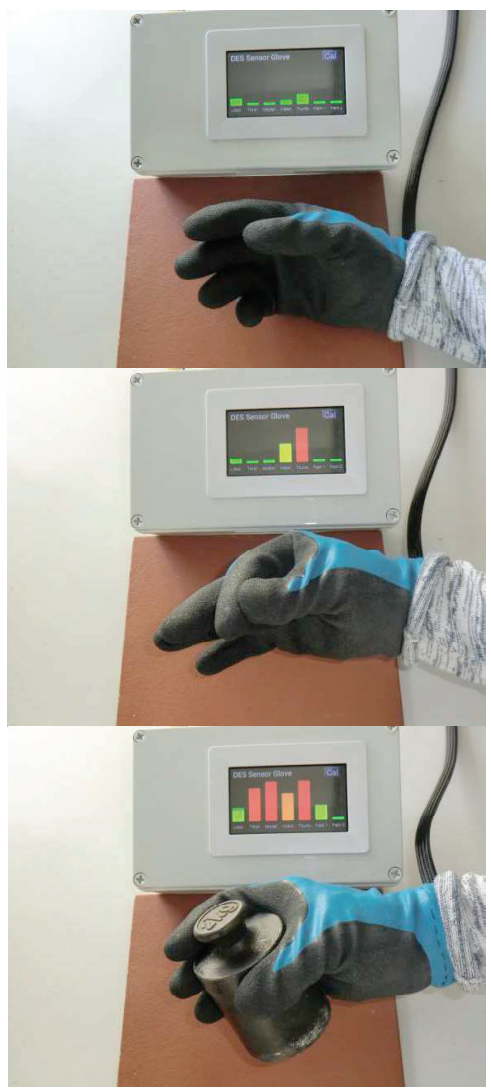

Abb. 15. Darstellung der Kapazitätserhöhung der 7 Sensoren auf dem Handschuh bei verschiedenen Tätigkeiten: unbelasteter Zustand (oben), Zusammendrücken von Daumen und Zeigefinger (Mitte) und Greifen eines Gewichtes von 2 kg (unten)

Eine mögliche Anwendung besteht darin, bei manuellen Tätigkeiten die Greifkräfte zu messen, um Überlastungen der Hände zu vermeiden. Zwar sind die hier gezeigten Sensoren relativ empfindlich und damit auf eher geringe Greifkräfte ausgelegt, doch erlaubt das dargestellte Messprinzip der 
Drucksensoren auch eine Auslegung auf deutlich höhere Druckkräfte.

Ein weiteres Anwendungsgebiet der neuen Drucksensoren ergibt sich in der Automatisierungstechnik. Entsprechende Sensoren können auch an Greifwerkzeugen von Robotern installiert werden. Durch die permanente Druckmessung lässt sich die Greifkraft besser steuern. Auf diese Weise sollen Beschädigungen insbesondere an empfindlichen und filigranen Objekten vermieden werden.

Ein weiteres bedeutendes Anwendungsgebiet der elastomeren Drucksensoren besteht im Bereich der Mensch-Maschine-Schnittstellen. $\mathrm{Da}$ die Sensoren Fingerdrücke sehr empfindlich und genau erfassen können, lassen sich damit auch technische Funktionen steuern.
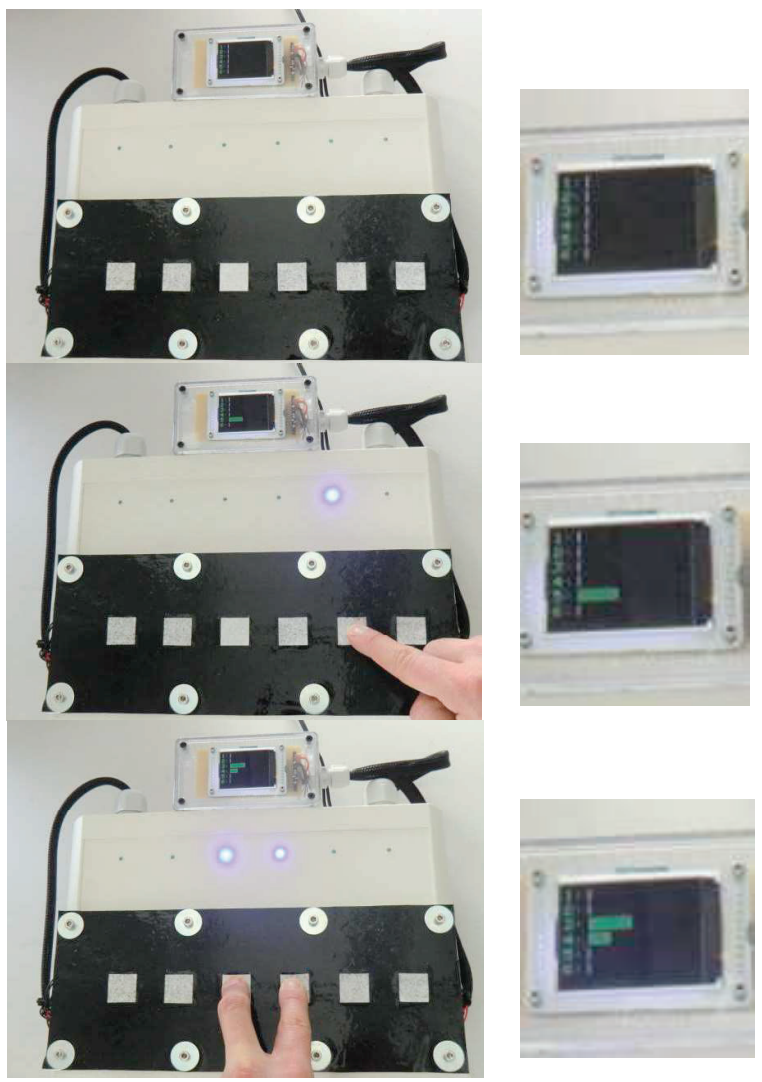

Abb. 16. Bedienoberfläche mit sechs Drucksensoren, zugehörigen LEDs sowie Balkenanzeige der ausgeübten Druckstärke (rechts) in verschiedenen Zuständen: kein Druck (oben), Druck auf das 5. Bedienfeld (Mitte) und unterschiedlicher Druck auf das 3. und 4. Bedienfeld (unten)

Um das Potential der Drucksensoren für Bedienelemente zu prüfen, wurde ein entsprechender Demonstrator aufgebaut, der in Abb. 16 dargestellt ist. $\mathrm{Er}$ enthält eine schwarze Bedienoberfläche mit insgesamt sechs hellen Bedienfeldern. Unter jedem Bedienfeld mit $20 \mathrm{~mm}$ x $20 \mathrm{~mm}$ Fläche befindet sich ein Drucksensor, der die Stärke des Fingerdruckes über die Messung der Kapazität quantitativ erfasst.

Die gemessenen Kapazitäten können dazu genutzt werden, um technische Vorgänge mit dem Finger zu steuern. Im dargestellten Demonstrator der Bedienoberfläche werden hier beispielhaft sechs LEDs in ihrer Helligkeit verändert. Zur quantitativen Anzeige werden die Kapazitäten wiederum als Balken auf einem Display angezeigt.

In Abb. 16 ist im oberen Bild der Zustand ohne Betätigung eines Bedienfeldes dargestellt. Im mittleren Bild wird mit einem Finger auf das fünfte Bedienfeld gedrückt. Entsprechend der Druckstärke leuchtet darüber eine blaue LED auf. Außerdem ist im rechten kleineren Bild das Display mit dem entsprechenden Balken, der die Kapazität des fünften Bedienfeldes anzeigt, vergrößert dargestellt. Im unteren Bild in Abb. 16 wird schließlich die simultane Betätigung von zwei Bedienfeldern gezeigt. Dabei wird mit zwei Fingern mit unterschiedlicher Stärke auf die beiden Bedienfelder 3 und 4 gedrückt. Demensprechend leuchten die zugehörigen LEDs verschieden stark auf. Auch die Balken auf dem Display zeigen die unterschiedliche Druckstärke an.

Damit wird deutlich, dass die neuen Drucksensoren nicht nur zur Erfassung von Druckkräften von Fingern in flexiblen Umgebungen wie einem Handschuh genutzt werden können. Im Bereich der MenschMaschine-Schnittstellen lassen sich solche Sensoren auch für die Steuerung von technischen Funktionen einsetzen. Dies ist insofern besonders interessant, weil auch hier die entsprechenden Bedienelemente in flexible Strukturen integriert werden können.

\section{Resümee}

Zur Detektion von Fingerdrücken eignen sich besonders flexible, weiche und empfindliche Drucksensoren. Um solche Sensoren zu realisieren, wurden zunächst kapazitive Sensormatten aus verschiedenen Elastomerkomponenten entwickelt. Diese Sensormatten bestehen aus zwei Elastomerprofilen, zwischen denen sich eine Elastomerfolie befindet. Bei der Druckbelastung dringen die Profile ineinander ein und dehnen die Folie. Auf diese Weise kommt es zu einer starken Kapazitätserhöhung. Ein weiterer dafür wesentlicher Effekt ist die Annäherung der Elektrodenschichten auf den Profilen und in der Zwischenfolie.

Dieses grundlegende Sensorprinzip wurde in verschiedenen Versionen realisiert. Die auf 
diese Weise aufgebauten Sensormatten zeigen sehr unterschiedliche Charakteristiken der Kennlinie der Kapazität in Abhängigkeit von der Druckbelastung. Zur Beeinflussung dieser Kennlinie sind verschiedene Design- und Materialparameter verfügbar. Ein wesentlicher Einflussfaktor ist die Form der eingesetzten Profile. Darüber hinaus spielen das Design und die Anordnung der Elektrodenschichten in der Sensormatte eine entscheidende Rolle. Die starke Variabilität der Sensorkomponenten lässt es damit zu, die Kennlinie auf die jeweils gewünschten Anforderungen abzustimmen.

Damit ergeben sich weitreichende Potentiale der neuen Sensoren zur Detektion von Fingerdrücken. Dies wurde anhand von zwei Anwendungsbereichen dargestellt. Mit Drucksensoren an einem Handschuh lassen sich Greifkräfte und auch die Druckverteilung auf der Hand quantitativ erfassen. Dies kann der Arbeitssicherheit dienen, um Überlastungen bei manuellen Tätigkeiten zu vermeiden. Eine entsprechende Sensorik kann ebenso Greifvorgänge von Robotern steuern, um Beschädigungen an den zu greifende Objekten zu verhindern.

Ein zweites mögliches Anwendungsfeld der neuen Sensoren zur Detektion von Fingerdrücken sind Mensch-MaschineSchnittstellen. Durch die Ausübung eines definierten Fingerdrucks lassen sich technische Funktionen stufenlos steuern. Auch dies wurde an einem Demonstrator mit der Beeinflussung der Helligkeit von LEDs gezeigt. Prädestiniert ist die flexible Drucksensorik besonders für den Einsatz auf nachgiebigen Unterlagen.

\section{Danksagung}

Für die Unterstützung der Arbeiten im Zentrum Smart Materials wird dem Bayerischen Staatsministerium für Wirtschaft und Medien, Energie und Technologie nachdrücklich gedankt.

\section{Literatur}

[1] F. Carpi, D. De Rossi, R. Kornbluh, R. Pelrine, P.Sommer-Larsen: Dielectric Elastomers as Electromechanical Transducers, Elsevier, 2008

[2] www.stretchsense.com

[3] S.C.B. Mannsfeld et al.: Highly Sensitive Flexible Pressure Sensors with Micro-structured Rubber Dielectric Layers, Nature Materials 9 (2010) 859-864

[4] H. Vandeparre, D. Watson, S. P. Lacour: Extremely robust and conformable capacitive pressure sensors based on flexible polyurethane foams and stretchable metallization, Applied Physics Letters 103 (2013) 204103
[5] T. Nakamura, Y. Inoue, D. Kim, N. Matsuhisa, T. Yokota, T. Seketani, T. Someya, M. Sekino: Basic characteristics of implantable flexible pressure sensor for wireless readout using MRI, Conf Proc IEEE Eng Med Biol Soc. (2014) 233841

[6] D. Kwon, T.-I. Lee, M. S. Kim, S. Kim, T.-S. Kim, I. Park: Porous dielectric elastomer based ultrasensitive capacitive pressure sensor and its application to wearable sensing device, Proc. Transducers Conf., Anchorage, Alaska, (2015) 299-302

[7] B.-Y. Lee, J. Kim, H. Kim, C. Kim, S.-D. Lee: Low-cost flexible pressure sensor based on dielectric elastomer film with micro-pores, Sensors and Actuators A 240 (2016) 103-109

[8] H. Böse, E. Fuß: Novel dielectric elastomer sensors for compression load detection, Proc. SPIE Vol. 9056 (2014) 905614 1-13

[9] H. Böse, F. Hofmann, T. Hassel, E. Fuß: Flexible Drucksensoren aus Dielektrischen Elastomeren. Konferenzband Sensoren und Messsysteme, Nürnberg, 03.-04.06.2014, VDEVerlag (2014), ISBN 978-3-8007-3622-5

[10] H. Böse, E. Fuß, P. Lux: Influence of design and material properties on the performance of dielectric elastomer compression sensors, SPIE Vol. 9430 (2015) 943079 1-12

[11] H. Böse, E. Fuß, J. Ehrlich: Capacitive sensor mats for pressure detection, Proc. SENSOR (2015) 55-60, AMA Conferences 19.21.05.2015, Nuremberg, Germany, DOI 10.5162/sensor2015/A2.2 